\title{
The Use of Application Scanners in Software Product Quality Assessment ${ }^{*}$
}

\author{
Stefan Wagner \\ Institute of Software Technology \\ University of Stuttgart \\ Stuttgart, Germany \\ stefan.wagner@informatik.uni-stuttgart.de
}

\begin{abstract}
Software development needs continuous quality control for a timely detection and removal of quality problems. This includes frequent quality assessments, which need to be automated as far as possible to be feasible. One way of automation in assessing the security of software are application scanners that test an executing software for vulnerabilities. At present, common quality assessments do not integrate such scanners for giving an overall quality statement. This paper presents an integration of application scanners into a general quality assessment method based on explicit quality models and Bayesian nets. Its applicability and the detection capabilities of common scanners are investigated in a case study with two open-source web shops.
\end{abstract}

\section{Categories and Subject Descriptors}

D.2.9 [Software Engineering]: Management-Software Quality Assurance

\section{General Terms}

Security, measurement

\section{Keywords}

Application scanner, quality assessment, Bayesian net, quality model

\section{INTRODUCTION}

Continuous quality control means to assess and improve software quality almost continuously, i.e., on an hourly or daily basis. This allows the developers to detect quality defects and to remove them early after their introduction into the system, which avoids a general quality decay and far higher costs in later phases in the software's life cycle. These

\footnotetext{
*This work has partially been supported by the German Federal Ministry of Education and Research (BMBF) in the project QuaMoCo (01 IS 08023B).
}

Permission to make digital or hard copies of all or part of this work for personal or classroom use is granted without fee provided that copies are not made or distributed for profit or commercial advantage and that copies bear this notice and the full citation on the first page. To copy otherwise, to republish, to post on servers or to redistribute to lists, requires prior specific permission and/or a fee.

WoSQ'11, September 4, 2011, Szeged, Hungary.

Copyright 2011 ACM 978-1-4503-0851-9/11/09 ...\$10.00. benefits, however, come at the cost that quality assessments need to be done often and hence are elaborate. Therefore, automation and good tool support is necessary to employ continuous quality control in practice [10].

\subsection{Problem Statement}

Software product quality assessments need to cover a large variety of topics including security. The assessment of product security is - as all quality analyses - elaborate. Hence, also for security, automation is necessary for practical adoption. In quality assessments automation relies to a large degree on automated static analysis. Static analysis, however, can only assess security partial. Dynamic analyses are needed to complement the static ones. Most existing automatic dynamic analyses for security are not integrated into product quality assessment methods. Instead, dynamic analysis tools, mostly so-called application scanners, are used solely for analysing the security of networks and hosts.

\subsection{Research Objective}

Similar to static analysis tools, there is a a plethora of tools for automatic dynamic security analysis. Especially application scanners are available commercially as well as open source. Those tools scan the executing application automatically for vulnerabilities and hence are a promising addition to static analysis. Our overall objective is to investigate the available tools and the kinds of vulnerabilities they detect to define how these tools should be integrated in a general quality assessment.

\subsection{Contribution}

We employ an existing quality assessment method based on explicit quality models and Bayesian nets and extend it by defining how application scanners can be used in the assessment. This extended method is performed using three well-known open source application scanners (w3af, Wapiti, and Grendel Scan) on two open source web shops (PHP Shop and Zen Cart). We show the principal applicability of the method to these real-world applications and also find first indications that the scanners find different vulnerabilities and, hence, should be used in combination. Therefore, this paper is only a first step in the direction of the research objective.

\subsection{Context}

The approach is applicable in principle to any kind of software. Most application scanners focus on web applications at present. The used application scanners and study object are open source but in use in commercial contexts. 


\subsection{Outline}

We start by introducing application security scanners and especially the used scanners in section 2 . We then explain the quality assessment method based on an explicit quality model and Bayesian nets that we will use as an example method into which we integrate the scanner results in section 3. In section, we describe the design and results of the case study. Finally, we compare our results with related work (section 5) and give final conclusions (section 6).

\section{APPLICATION SECURITY SCANNERS}

We first give a general introduction into what application security scanners are and present three scanners that are also used in the case study in Section 4.

\subsection{General}

In general, an application scanner is a software that performs automatic penetration testing. Most scanners use a set of common patterns of inputs that they send to the application and decide, based on the output, whether there is a vulnerability that might be exploited. In addition, they have many possibilities to configure the penetration tests so that they fit to the system under analysis. Most application scanners concentrate on web applications as these are most exposed to attacks. Black et al. define in [7] a Web application security scanner as an "automated program that searches for software security vulnerabilities within web applications".

There are several groups that work on specific application scanners (e.g., $[4,19,20])$ in order to either find new vulnerabilities or improve the detection of vulnerabilities. There are also specialised tools that dynamically and (partly) statically detect specific vulnerabilities [3]. However, these different tools have not been compared and analysed w.r.t. their usage in product quality assessment. We discuss three common open-source scanners in the following.

\section{2 w3af}

The Web Application Attack and Audit Framework (w3af) provides a framework as well as a complete graphical and command-line interface to run application scans and view results. The framework provides simple wrappers for HTTP communication, web services, sessions, and HTML parsing. It also contains many plugins that implement scanning and testing an application. It is written in Python and is available at http://w3af . sourceforge.net/.

\subsection{Wapiti}

The Web application vulnerability scanner / security auditor (Wapiti) is a command-line tool that scans the web pages of an application and identifies scripts and forms to inject data. Using these scripts and forms it acts like a fuzzer and injects payloads to see if a script is vulnerable. Wapiti is developed in Python. It is available at http: //wapiti.sourceforge.net/.

\subsection{Grendel-Scan}

Grendel-Scan is a web application security testing tool that also provides a graphical user interface. It contains an automatic application scanner that detects common web application vulnerabilities. It is written in Java and is available at http://www.grendel-scan.com/.

\section{QUALITY ASSESSMENT METHOD}

Quality assessment is the part in quality control that compares the actual state of an application with its requirements. It evaluates if and how well the software fits to what was intended. There are various ways to perform this assessment and the major difficulty lies in combining the various quality assurance results and measures to a common quality statement. In the project Quamoco ${ }^{1}$, we developed such a quality assessment method. One specific instance of this method uses Bayesian nets to describe the uncertainties in the results and measures as well as to calculate a quality statement. In the following, we propose how application scanners can be integrated in the method for a substantial security assessment.

\subsection{Quamoco}

In the project Quamoco, we develop a quality model with a corresponding quality assessment method that has the aim to facilitate continuous improvement based on objective, quantitative feedback [21]. It has its origins in the Quality Improvement Paradigm [5] and the Goal/Question/Metric (GQM) approach [6]. We built one specific instance using Bayesian nets as a means for analysing assessment results [26] that was specifically aimed at using activity-based quality models [12].

We give a brief overview on the quality models developed in Quamoco and describe the assessment method from [26] adapted to the Quamoco quality models.

\subsection{Quamoco Quality Models}

In general, there are two main uses of quality models in a software project: (1) as a basis for defining quality requirements and (2) for defining quality assurance techniques and measurements for the quality requirements. The quality models developed in Quamoco advance existing quality models as they combine the practically shown advantages of different models $[12,17,25,28]$. The idea is to use not only high-level "-ilities" for defining quality but instead to break it down into detailed factors and their influence on quality attributes. The quality attributes we use in this paper are the activities performed on and with the system, which are derived from activity-based quality models [12]. In the area of security, we use a hierarchy of attacks as activities [27]; in this case activities that should be prevented.

We developed an explicit meta-model in Quamoco that defines the quality model elements and their relationships. Five elements of the meta-model are most important in the context of this paper: entity, property, measure, impact, and activity. An entity can be any thing, animate or inanimate, that has an influence on the software's quality, e.g., the source code of a PHP function or an HTML form. These entities are characterised by properties such as structuredness or conformity. The combination of an entity and a property is called a factor. These factors are measurable either by automatic measurement or by manual review. This is specified in the measures for a factor.

Entities as well as activities are organised in hierarchies. An influence of a factor is specified by an impact. We concentrate on the influences on attack activities, for example, $S Q L$ injection or password brute forcing. The impact on an activity can be positive or negative.

\footnotetext{
${ }^{1}$ http://www.quamoco.de/
} 


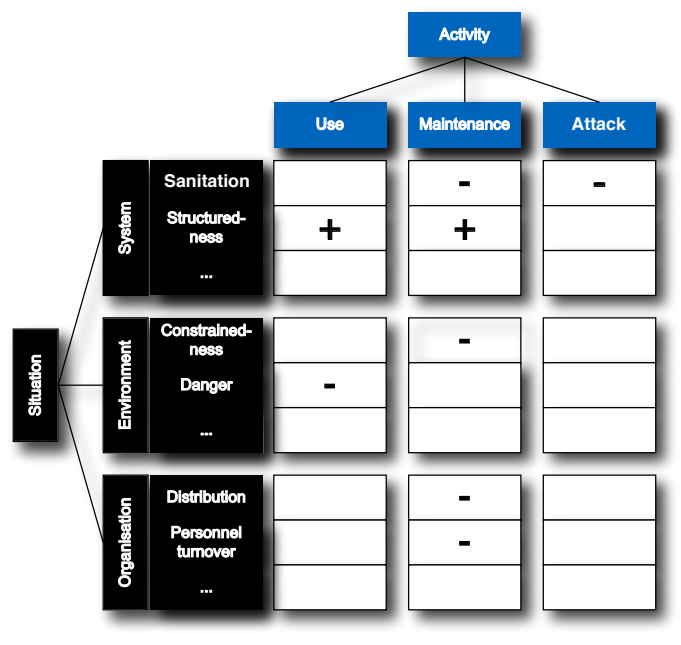

Figure 1: High-level view on an activity-based quality model as a matrix (based on [26])

The two hierarchies, the factor tree and the activity tree, together with the impacts of the facts on the activities can be visualised as a matrix (Figure 1). The factor tree is shown on the left, the activity tree on the top. The impacts are depicted by entries in the matrix where a "+" denotes a positive and a "_" a negative impact. The associations between factors in the factor tree denote a "kind-of" relationship.

\subsection{Bayesian Nets}

Bayesian nets are a means for modelling uncertain relationships that can be simulated and that predicts probable outcomes. They are a modelling technique that can represent causal relationships based on Bayesian inference. They are represented as a directed acyclic graph with nodes for uncertain variables and edges for directed relationships between the variables. This graph models all the relationships abstractly.

For each node or variable there is a corresponding node probability table (NPT). These tables define the relationships and the uncertainty in these variables. For each state of each variable, the probability that the variable is in this state is specified. If there are parent nodes, i.e., a node that influences the current node, these probabilities are defined in dependence on the states of these parents. A complete Bayesian net allows the forward and backward calculation for different scenarios based on observations or desired outcomes.

\subsection{Steps}

The assessment method [26] consists of four steps for building a Bayesian net derived systematically from a Quamoco quality model. The resulting Bayesian net contains three types of nodes:

- Activity nodes that represent activities from the quality model

- Factor nodes that represent factors from the quality model

- Measure nodes that represent indicators for activities or factors
We need four steps to derive these nodes from the information of the quality model.

1. We identify the relevant activities with measures based on the assessment goal. We use GQM [6] to structure that derivation. We first define the assessment goal, for example, optimisation of security assurance, which leads to relevant activities, such as attack. This is refined by stating questions that need to be answered to reach that goal.

2. Influences by sub-activities and factors are identified. This step is repeated recursively for sub-activities. The resulting factors together with their impacts are modelled.

3. Suitable measures for the factors are added.

4. The node probability tables (NPT) are defined to reflect the quantitative relationships. This includes defining node states as well as filling the NPT for each node. The activity and factor nodes are usually modelled as ranked nodes, i.e., in an ordinal scale. Having that, the Bayesian net can be used for simulation by setting values for any of the nodes.

The definition of NPTs is the most complicated part in building Bayesian nets. The approach by Fenton, Neil and Galan Caballero [14] simplifies that by approximating the specific values in an NPT by general distributions or expressions. They formalise the behaviour observed with experts that have to estimate NPTs, who usually estimate the central tendency or some extreme values based on the influencing nodes. The remaining cells of the table are then filled accordingly. For example, it renders it possible to model the NPT of a node by a weighted mean over the influencing nodes.

In general, the NPTs of the measure nodes are defined using either common industry distributions or information from company-internal measurements. The influence of the activity or factor node it belongs to can be modelled in at least two ways: (1) partitioned expressions and (2) arithmetic expressions. The latter describes a direct arithmetical relationship from the level in the activity or factor node to the measure. Using a partitioned expression, the additional uncertainty can be expressed by defining probability distributions for each level of the activity or factor node.

\subsection{Integration of Application Scanners}

Application scanners provide findings of probable vulnerabilities for the analysed software. We can use them as measures for factors. Hence, the integration of application scanners affects the steps 3 and 4 of the assessment method. We define measure nodes that correspond to scanner findings. All scanners classify the found vulnerabilities into different types. Each vulnerability type forms a measure. These measures are matched to existing factors or new factors are generated enriching the knowledge about how to develop secure software applications.

For example, an application scanner might detect buffer overflows if the software is configured to return error pages. The assessment method user would create a measure node Buffer Overflow Error Page that represents the findings of the scanner. The quality model already contains a factor Confinement of Buffer, which specifies that the limits 
of buffers are respected. This factor is represented in the Bayesian net as a factor node and the assessor adds an influence to the measure node.

The factors that are measured by application scanners can have an impact on a very specific attack or in general ease attacking. This is reflected by the hierarchy level of the attack that has the impact. A general impact goes to a more generic attack in the activity hierarchy. For the example of the buffer overflow, the impact might be on the attack Forced Integer Overflow that represents the setting of a controllable integer value to an unexpected value.

For measures from static analysis, we calculate densities to reflect how large the problems are in relation to the software size. As each found vulnerability can potentially corrupt the complete application, we use a simpler yes/no voting. If there is at least one vulnerability of a type, the measure has the value yes. For example, if the scanner detects at least one buffer overflow error page, the assessor sets the observation of the measure node to yes. The NPT in the measure node is modelled by a partitioned expression. In the buffer overflow example, if Confinement of Buffer is in the state high, Buffer Overflow Error Page is in the state no and vice-versa. The expression should also add an uncertainty range depending on how well the measure indicates the factor.

If we employ more than one scanner, we can run into the problem that the scanners do not agree on the detection of specific vulnerabilities. We prefer a pessimistic assessment - possibly worse than it actually is - and hence vote yes if at least one scanner reports a vulnerability.

\section{CASE STUDY}

The case study shows the applicability of the method and to a smaller degree the detection capabilities of application scanners. We define the study design, describe the used study objects, and show and discuss the results.

\subsection{Study Design \& Procedure}

The aim of this case study is a proof-of-concept that analyses the method's applicability to real-world software. In particular, we are interested in the effort needed to incorporate and use the scanners as well as if they give useful results. Furthermore, the execution time for analysis should be short enough to be able to run the scanners often, e.g., on a daily basis. This leads to our first research question:

$\mathrm{RQ}$ 1. Is the assessment method applicable to realistic software systems?

Moreover, we investigate if common scanners are comparable in terms of the vulnerabilities they detect. The experience with static analysis has shown that different tools detect partly different classes of defects. If this is not the case, we could resort to just one tool in quality control, which would reduce our effort considerably. Hence, our second research question asks for the differences in vulnerability detection:

RQ 2. Are there differences between the detection capabilities of different application scanners?

We analyse both questions by applying 3 widely known open-source application scanners (see Section 2) to 2 opensource web shops. We install both web shops with their standard installation and run each scanner on each web shop. The scanners are configured to reasonable settings w.r.t. the study objects. For example, attacks specifically for Microsoft SQL Server make no sense as a MySQL database system is used by the study objects.

The vulnerabilities found by all scanners are partitioned into classes that stem from the types of vulnerabilities found by the scanners. The classes are used in the quality assessment method to make a quality statement about the study objects. The Bayesian net for that is built using the tool AgenaRisk. We analyse this application of the method qualitatively to answer RQ 1 . Then we compare the results of all three scanners separately and compare their findings for answering RQ 2. The comparison analyses to what degree there are overlaps in the found classes of vulnerabilities. The vulnerabilities are not checked for false positives.

\subsection{Study Objects}

The study objects are two different web shops, one - Zen Cart - a large application, which is also the most popular of this kind on sourceforge. The other application - PHP Shop - is simple and small in comparison to Zen Cart. Hence, in the case selection, triangulation is used as far as possible. Detailed descriptive information about both study objects is given in Table 1 .

Table 1: Information about the study objects

\begin{tabular}{lrr}
\hline & PHP Shop & Zen Cart \\
\hline Language & PHP & PHP/Perl \\
1. Release & 1999 & 2004 \\
Database & MySQL & MySQL \\
Used Version & 0.8 .1 & 1.3 .8 \\
SLOC & 8,052 & 73,001 \\
Downloads & 53,000 & 625,000 \\
\hline
\end{tabular}

Both applications were installed in the standard Apache web server available in Mac OS X and connected to a local MySQL installation as database management system. As far as possible all configuration were left with the default values.

\subsection{Results}

As a result for the applicability of the approach, we describe the concrete application together with our experiences. We start with the first step of our assessment approach and identify the relevant activities and corresponding measures. We analyse security, in particular the risk of vulnerabilities in the system. The risk can be the basis for deciding whether further security improvements needs to be employed. Therefore, the goal is "Planning of further security improvements". For security improvements, attacks on the system need to be confounded. Hence, the activity Attack needs to be analysed. We derive the question "How many vulnerabilities are there in relation to the software size?". For the security improvement planning, it is not only important how many vulnerabilities there are but also whether this number is in a reasonable relation to the system size. It might be economically inadvisable to invest in removing all vulnerabilities. The corresponding metric vulnerability density that measures the number of vulnerabilities by source code size in KSLOC can be directly derived from the question.

In the second step of the assessment method, we build the Bayesian net. The selection of the nodes in the study 
is driven by the detection possibilities of the used scanners. There is the top-level activity Attack that we measure by the above derived vulnerability density. It has a direct impact from Visibility of Public Code Comment that describes that it is easier to attack if there are code comments visible to the public. Then, we break Attack down to Probabilistic Techniques, Injection, and Exploitation of Trusted Credentials. These are further refined into Password Brute Forcing, Script Injection, SQL Injection, Cross site Request Forgery, and Session Credential Falsification Through Prediction. Figure 2 shows in the top left the activity tree as represented in the Bayesian Network.

We include 6 impacts on these activities. The impacts are chosen so that their corresponding factors can be measured by the investigated application scanners. The factors used are:

- Completeness of Password Change: Any implementation of changing user passwords is also responsible for the quality of that password to avoid password brute forcing. If such a check is missing, we consider the implementation to be incomplete.

- Sanitation of Dynamic Web Page: If a web application does not sufficiently sanitise the data it is using in output, arbitrary content, including scripts, can be included by attackers.

- Sanitation of SQL Statement: Analogously to dynamic web pages, the used SQL statements need to be sanitised to avoid unwanted changes or reads to the database.

- Visibility of Public Code Comment: Comments in HTML or Java Script code visible to the public may give attackers information they can exploit.

- Authenticity of Request: The application needs to be able to undoubtedly decide on the authenticity of a request. If this is not the case, Cross Site Request Forgery is possible.

- Uniqueness of Session ID: Each session needs a unique ID that cannot easily be guessed. Otherwise an attacker may predict an ID and gain access to the application

In the fourth step of the approach, measures are defined for all impacts. The measures here are derived from the vulnerabilities identifiable by the scanners and are attributable to the application - as opposed to the environment. The final topology of the Bayesian net is shown in Figure 2. Overall, building the Bayesian net took less than a day.

The execution of the scanners took between several minutes (PHP Shop) and several hours (Zen Cart) on a current MacBook Pro that runs both the web server and the scanners. The found vulnerabilities are shown in Table 2. Waipiti did not find any vulnerabilities in both cases. We analysed its execution in detail to avoid any misconfigurations, but it seems that it is not able to detect problems in the analysed software. Most vulnerabilities were detected by Grendel Scan, 3 vulnerabilities were reported by w3af.

This information was then used in the Bayesian net to assess the quality of the two applications. Two vulnerability classes from Table 2, the input/output flows and unidentified vulnerabilities, were not further used because they cannot
Table 2: The vulnerabilities found in the scans. The characters $\mathrm{A}-\mathrm{C}$ denote which scanner found the vulnerability: $A=w 3 a f, B=$ Wapiti, $C=$ Grendel Scan.

\begin{tabular}{lcc}
\hline Vulnerability & PHP Shop & Zen Cart \\
\hline Duplicate Session ID & $\mathrm{C}$ & $\mathrm{C}$ \\
Potential CSRF & $\mathrm{A}, \mathrm{C}$ & $\mathrm{C}$ \\
SQL Injection & & $\mathrm{A}, \mathrm{C}$ \\
Code Comments & $\mathrm{C}$ & $\mathrm{C}$ \\
Unidentified Vuln. & $\mathrm{A}$ & \\
Input/Output Flows & $\mathrm{C}$ & \\
\hline
\end{tabular}

be attributed to a specific product entity. The predicted vulnerability densities (vulnerabilities/KSLOC) of both applications are very close. The net calculated a mean of 0.0064 for PHP Shop (standard deviation 0.0028) and a mean of 0.0066 for Zen Cart (standard deviation 0.0028).

\subsection{Discussion \& Threats to Validity}

The assessment method including application scanners is applicable to the real-world systems we analysed. Yet, the results are close for both systems and the correctness of the results cannot be validated as we have no data about the real vulnerability density. Nevertheless, the effort for performing the assessment is reasonable. The setup of the scanners and a corresponding test environment is more demanding than the subsequent analysis using the Bayesian net. Altogether it took only a few days to set up the analysis. Also the time needed for running the scanners is promising and allows them to be included in continuous quality control.

An important decision in modelling application security is the border between the application that is analysed and its environment. For example, is the application responsible for passwords that are not prone to brute force attacks? In the case study we made subjective choices and for the example of passwords specified that the application has a partial responsibility.

The answer to RQ 2 is more clear as the found vulnerabilities differ between the used scanners. Wapiti did not find a single vulnerability, Grendel Scan found 8 and w3af found 3 vulnerabilities. For potential cross site request forgery in PHP Shop and SQL injection in Zen Cart both scanners had findings. However, only Grendel Scan detected a potential CSRF in Zen Cart. All these differences indicate that there are significant differences between the detection capabilities of different scanners.

As this is only a first, explorative study on the use of application scanners in quality assessment, there are various threats to the validity of the results. The internal validity is threatened because there were several subjective decisions in building the Bayesian net. We mitigated this threat by using comparable decisions as for static analysis. Furthermore, we did not check whether the found vulnerabilities are actual problems in the software. This especially affects RQ 2, because the results might be misleading. The external validity is also limited as we only analysed two applications and three scanners, which are all open source. For more reliable results, especially for the detection capabilities, we need to run larger studies that also involve commercial applications and scanners. 


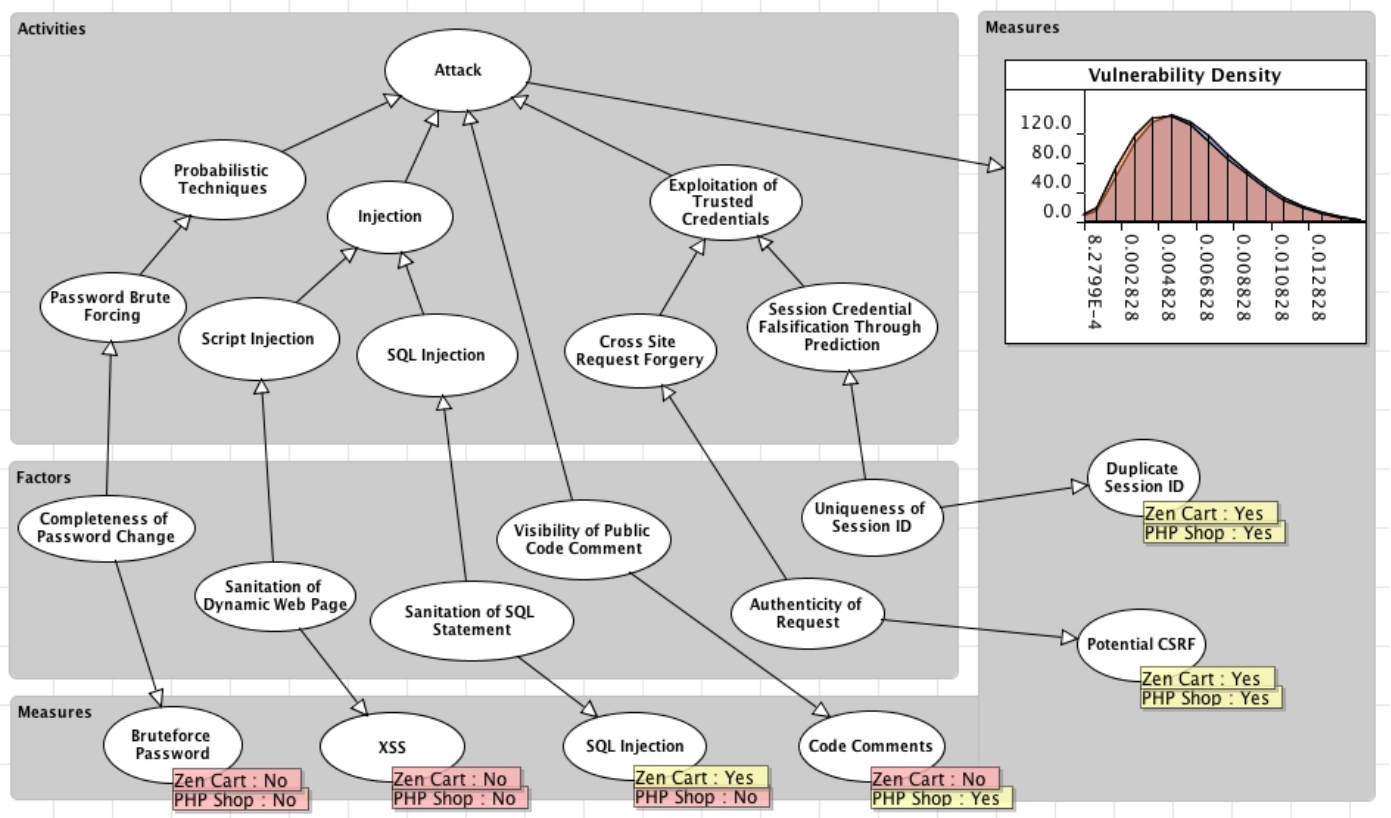

Figure 2: The Bayesian net for the analysis from AgenaRisk

\section{RELATED WORK}

We discuss quality models, guidelines and measures and especially several security assessment approaches.

There is a wide variety of quality models. Deissenboeck et al. [11] differentiate between quality definition models and quality assessment models. The former is a specification of what constitutes quality in a software system, the latter describes how a software system's quality can be assessed according to specific rules. In the area of software security, security pattern collections are an example of quality definition models, e.g., [18].

Quality definition models are either general but too abstract for a concrete use in assessing software quality (e.g., ISO 9126) or specialised for a specific quality attribute and hence difficult to integrate into general quality assessments [12]. In [12], Deissenboeck et al. propose a quality model (ABQM) that tackles this problem by breaking quality attributes into entities, their properties, and their influence on activities. In [27] we used the ABQM approach for modelling security but with a focus on security requirements.

Quality guidelines are developed by various companies and organisations and usually include technical aspects that have to be taken into account. For example, the Common Criteria catalog (CC) [9] and the German BSI ITGrundschutz Manual [13] describe security requirements. Usually guidelines do not give rationales [12]. Hence, they do not guide through a structured process, are often read once and followed in a sporadic manner only [8]. Furthermore, it is often not checked whether guidelines are followed or not $[12]$.

Common metric-based/stochastic approaches describe quality by measurable concepts that imply strong assumptions. While for some quality attributes, those assumptions are stable for others, such as security, the assumptions are changing fast [1]. Due to their single-value representation, met- rics often do not explain how system properties influence the quality related activities that are performed with the system [12]. Hence, metrics are not well established for security [2] and unstable due to fast variation of the security underlying "physics" (i.e., the IT system) [1].

Artsiom et al. [29] propose an security assessment method that has similarities to the method in this paper. It also defines metrics and aggregates them to quality attributes. This method, however, uses "-ilities" similar to ISO 9126 that have several well-known problems. Moreover, they concentrate on the architecture of the software (white-box view) whereas this paper focuses on testing by application scanners (black-box view).

Frigault et al. [16] use Dynamic Bayesian Networks to investigate the security of networked systems. Their focus is more on the combined effects of different vulnerabilities as opposed to a complete quality statement for the system incorporating scan results.

There are several so-called scoring systems that evaluate vulnerabilities in applications. The most advanced scoring system is the Common Vulnerability Scoring System (CVSS) $[15,22]$. It provides a set of metrics and corresponding equations that combine these metrics with weights to provide a score for a vulnerability. It considers the constraints as well as the impacts of a vulnerability, but does describe how to find vulnerabilities and how to relate the results to a general quality assessment.

Recently the Common Weakness Scoring System (CWSS) [23] was released that analyses weaknesses in a software system and assigns scores for it for prioritising the weaknesses. One part of the scoring is the technical impact. Hence, there are similarities to the Quamoco quality model, which we should exploit in the future. By itself, the CWSS describes not how it fits into an overall quality assessment.

The Open Web Application Security Project (OWASP) is a non-commercial initiative to develop guidelines and stan- 
dards for the security of web applications. Their OWASP Application Security Verification Standard 2009 (ASVS) [24] defines 4 security verification levels that describe what has to be done to provide appropriate security for an application. The developer of an application decides on its criticality and the standards gives the corresponding verification requirements that have to be met. This ranges from mostly automatic analysis to complete manual code reviews. A level is reached if all these requirements are checked. It does not contain more fine-grained evaluations and it is also not set into the context of a general quality assessment.

\section{CONCLUSIONS}

We summarise the contribution of this paper and discuss directions for future research.

\subsection{Summary}

Application security scanners, as employed in the area of web applications, are one promising possibility to automate the assessment of software application security. This automation could then be used in product quality assessments in the context of continuous quality control. However, the usage of application scanners in this kind of quality assessment has not been investigated so far.

We provide a first step to incorporate application scanners into quality assessment by extending an existing method based on explicit quality models and Bayesian nets. In the Quamoco quality models, measures are defined to make use of the scanning results. It is also defined how these results can be further used for a general quality statement.

We show in a case study how three open source application scanners can be used in the quality assessment of open source web shop applications. We found that the method is applicable in principle and that the detection capabilities of the scanners differ. Moreover, the needed time for performing the scans is promising for their inclusion into continuous quality control.

\subsection{Future Work}

A threat for the case study is that only three scanners are used. We plan to evaluate more application scanners, especially tools developed commercially. For a more reliable result we also plan to investigate further cases involving software developed in industry for which we also analyse the false positive rate of scanners.

The assessment method will be extended to be able to handle false positives explicitly. The found differences between scanners might also be an indication for false positives and then the method could mitigate that by a larger weight for vulnerabilities that are found by more than one scanner. Finally, a study involving the combination and comparison with static analysis would show the strength and weaknesses of both approaches.

\section{Acknowledgements}

I am grateful to Elmar Juergens for helpful suggestions on the manuscript.

\section{REFERENCES}

[1] M. D. Aime, A. Atzeni, and P. C. Pomi. The risks with security metrics. In Proc. 4th ACM Workshop on Quality of Protection (QoP'08), pages 65-70. ACM Press, 2008.
[2] A. Atzeni and A. Lioy. Why to adopt a security metric? a brief survey. In Quality of Protection, volume 23 of Advanced in Information Security, pages 1-12. Springer, 2006.

[3] D. Balzarotti, M. Cova, V. Felmetsger, N. Jovanovic, E. Kirda, C. Kruegel, and G. Vigna. Saner: Composing Static and Dynamic Analysis to Validate Sanitization in Web Applications. In Proc. IEEE Symposium on Security and Privacy, pages 387-401. IEEE Computer Society, 2008.

[4] D. Balzarotti, M. Cova, V. Felmetsger, and G. Vigna. Multi-module vulnerability analysis of web-based applications. In Proc. ACM Conference on Computer and Communication Security (ACM CCS). ACM Press, 2007.

[5] V. Basili and H. Rombach. The TAME project: Towards improvement-oriented software environments. IEEE Transactions on Software Engineering, 14(6):758-773, 1988.

[6] V. R. Basili, G. Caldiera, and H. D. Rombach. Goal question metric paradigm. In J. C. Marciniak, editor, Encyclopedia of Software Engineering, volume 1. John Wiley \& Sons, 1994.

[7] P. Black, E. Fong, V. Okun, and R. Gaucher. Software assurance tools: Web application security scanner functional specification version 1.0. Special Publication 500-269, National Institute of Standards and Technology, 2008.

[8] M. Broy, F. Deissenboeck, and M. Pizka. Demystifying maintainability. In Proc. Intern. Workshop on Software Quality (WoSQ '06), pages 21-26. ACM Press, 2006.

[9] CCRA. Common criteria for information technology security evaluation, version 3.1. http://www . commoncriteria.org, 2009.

[10] F. Deissenboeck, E. Juergens, B. Hummel, S. Wagner, B. Mas y Parareda, and M. Pizka. Tool support for continuous quality control. IEEE Softw., 25(5):60-67, 2008.

[11] F. Deissenboeck, E. Juergens, K. Lochmann, and S. Wagner. Software quality models: Purposes, usage scenarios and requirements. In Proc. 7th International Workshop on Software Quality (WoSQ 09). IEEE Computer Society, 2009.

[12] F. Deissenboeck, S. Wagner, M. Pizka, S. Teuchert, and J. F. Girard. An activity-based quality model for maintainability. In Proc. IEEE International Conference on Software Maintenance (ICSM 2007), pages 184-193. IEEE Computer Society, 2007.

[13] Federal Office for Information Security (BSI) in Germany. IT-Grundschutz Catalogues. https://www.bsi.bund.de/, 2007.

[14] N. E. Fenton, M. Neil, and J. G. Caballero. Using ranked nodes to model qualitative judgments in Bayesian networks. IEEE Transactions on Knowledge and Data Engineering, 19(10):1420-1432, 2007.

[15] FIRST. Common vulnerability scoring system (CVSS). http://www.first.org/cvss/cvss-guide.html, 2009.

[16] M. Frigault, L. Wang, A. Singhal, and S. Jajodia. Measuring network security using dynamic bayesian network. In Proc. 4th ACM Workshop on Quality of Protection (QoP '08), pages 23-30. ACM Press, 2008. 
[17] M. Grossmann. Towards an applicable software quality model for individual software projects. In Workshop-Band Softwarequalitätsmodellierung und -bewertung (SQMB '09). TU München, 2009.

[18] M. Hafiz, P. Adamczyk, and R. Johnson. Organizing security patterns. IEEE Softw., 24(4):52-60, 2007.

[19] Y.-W. Huang, C.-H. Tsai, D. Lee, and S.-Y. Kuo. Non-detrimental web application security scanning. In Proc. 15th International Symposium on Software Reliability Engineering (ISSRE 2004), pages 219-230. IEEE Computer Society, 2004.

[20] S. Kals, E. Kirda, C. Kruegel, and N. Jovanovic. Secubat: a web vulnerability scanner. In Proc. 15th International Conference on World Wide Web (WWW '06), pages 247-256. ACM Press, 2006.

[21] C. Lampasona, A. Trendowicz, M. Kläs, and J. Heidrich. Measurement-based software quality evaluation. In Tagungsband des DASMA Software Metrik Kongresses 2009. Shaker, 2009.

[22] P. Mell, K. Scarfone, and S. Romanosky. Common vulnerability scoring system. IEEE Security and Privacy, 4(6):85-89, 2006.

[23] Mitre. Common weakness scoring system (CWSS). http://cwe.mitre.org/cwss/.
[24] OWASP. OWASP application security verification standard 2009. http://www.owasp.org/.

[25] R. Plösch, H. Gruber, A. Hentschel, C. Körner, G. Pomberger, S. Schiffer, M. Saft, and S. Storck. The EMISQ method and its tool support-expert-based evaluation of internal software quality. Innovations in Systems and Software Engineering, 4(1):3-15, 2008.

[26] S. Wagner. A Bayesian network approach to assess and predict software quality using activity-based quality models. Information and Software Technology, 52(11):1230-1241, 2010.

[27] S. Wagner, D. Mendez Fernandez, S. Islam, and K. Lochmann. A security requirements approach for web systems. In Workshop Quality Assessment in Web (QAW 2009). 2009.

[28] S. Winter, S. Wagner, and F. Deissenboeck. A comprehensive model of usability. In In Proc. Engineering Interactive Systems 2007 (EIS '07, volume 4940 of $L N C S$, pages 106-122. Springer, 2007.

[29] A. Yautsiukhin, R. Scandariato, T. Heyman, F. Massacci, and W. Joosen. Towards a quantitative assessment of security in software architectures. In Nordic Workshop on Secure IT Systems (NordSec), October 2008. 